\title{
Molecular Basis of Medium Chain Acyl-Coenzyme A Dehydrogenase Deficiency An A to G Transition at Position 985 That Causes a Lysine-304 to Glutamate Substitution in the Mature Protein Is the Single Prevalent Mutation
}

Ichiro Yokota, ${ }^{*}$ Yasuhiro Indo, ${ }^{*}$ Paul M. Coates, ${ }^{\dagger}$ and Kay Tanaka*

*Department of Human Genetics, Yale University School of Medicine, New Haven, Connecticut 06510; and ${ }^{\dagger}$ Division of Gastroenterology and Nutrition, Department of Pediatrics, University of Pennsylvania School of Medicine,

Philadelphia, Pennsylvania 19104

\begin{abstract}
We sequenced polymerase chain reaction (PCR)-amplified variant medium chain acyl-CoA dehydrogenase (MCAD) cDNAs in cultured fibroblasts from three MCAD-deficient patients. In all three patients, an $A$ to $G$ transition was identified at position 985 of the coding region. Since no appropriate restriction sites for detecting this point mutation were found, we devised a PCR method that amplifies an 87-bp fragment from position 955 . In the $5^{\prime}$ primer encompassing positions 955 to 984, A-981 was artificially substituted with $C$. With the presence of C-981 and G-985, an Nco I restriction site is introduced in the mutant copies. When cDNA or genomic DNA from fibroblasts of nine MCAD-deficient patients were tested with this method, the copies from all of them were completely cleaved into two shorter fragments by Nco I, indicating their homozygosity for the $A \rightarrow$ G-985 transition. In contrast, the copies from all eight controls remained intact. Thus, this $A \rightarrow$ G-985 transition is the single prevalent mutation causing MCAD deficiency, a highly unusual. feature for any genetic disorder. The PCR/Nco I digestion method is suitable for the diagnosis of MCAD deficiency. (J. Clin. Invest. 1990. 86:1000-1003.) Key words: medium chain acyl-CoA dehydrogenase $\bullet$ point mutation
\end{abstract}

\section{Introduction}

Medium chain acyl-CoA dehydrogenase (MCAD) ${ }^{1}$ deficiency is an inborn error of fatty acid oxidation. Since it was first reported in 1982 (1), over 100 cases have been reported. Its incidence may be as high as 1 in 10,000 births (2). The typical symptoms of MCAD deficiency are fasting intolerance, vomiting, and episodes of lethargy and coma, accompanied by hypoketotic hypoglycemia and medium chain dicarboxylic acid-

Address requests for reprints to Dr. Kay Tanaka, Dept. of Human Genetics, Yale University School of Medicine, 333 Cedar Street, New Haven, CT 06510.

Received for publication 3 May 1990.

1. Abbreviations used in this paper: CRM, cross-reacting materials; IVD, isovaleryl-CoA dehydrogenase; MCAD, medium chain acyl-CoA dehydrogenase; $p$, precursor; PCR, polymerase chain reaction; SCAD, short chain acyl-CoA dehydrogenase.

J. Clin. Invest.

(c) The American Society for Clinical Investigation, Inc.

0021-9738/90/09/1000/04 \$2.00

Volume 86, September 1990, 1000-1003 uria. Unless appropriately treated, these acute episodes can be fatal. Until such an episode abruptly occurs, patients with MCAD deficiency can appear to be healthy. It now seems that some children who were previously diagnosed with sudden infant death syndrome have in fact died of MCAD deficiency (3).

MCAD catalyzes the dehydrogenation of acyl-CoA esters with 6 to 12 carbons (4), and is one of five analogous dehydrogenases that belong to the acyl-CoA dehydrogenase family (5). Other enzymes in this gene family are long chain, short chain (SCAD), and 2-methyl-branched chain acyl-CoA dehydrogenases, and isovaleryl-CoA dehydrogenase (IVD). Like the other four enzymes, MCAD is a tetrameric mitochondrial flavoenzyme (4). Its subunit is encoded in the nucleus and synthesized in the cytosol as a $49-\mathrm{kD}$ precursor ( $p$ ) that contains a 4-kD $\mathrm{NH}_{2}$ terminal sequence extension (leader peptide) that directs pMCAD to the mitochondria. After uptake by the mitochondria, the precursor subunit is proteolytically processed to the $45-\mathrm{kD}$ mature subunit and assembled into the tetrameric form (6). Recently, human and rat pMCAD cDNAs (7, 8 ) have been cloned and sequenced. The coding region of both human and rat pMCAD cDNAs is $1,263 \mathrm{bp}$ long, and encodes the 421 -amino acid precursor pMCAD $(7,8)$. The human and rat pMCADs share $86.5 \%$ identical amino acid residues. The $\mathrm{NH}_{2}$ terminus of mature human MCAD has not been determined. In rat, it is Lys- 26 of the precursor.

Our previous study of variant MCAD proteins in cultured skin fibroblasts from 13 patients with MCAD deficiency using $\left[{ }^{35} \mathrm{~S}\right]$ methionine labeling and immunoprecipitation revealed that, in all instances, the size of variant MCADs was comparable to normal, suggesting that each of the variant MCADs in these 13 cell lines contain a point mutation (9). The variant pMCAD was properly processed to the mature form in all cell lines (9).

In this paper, we report the results of a study of nine unrelated MCAD-deficient cell lines using the polymerase chain reaction (PCR), which revealed that all nine patients were homozygous for an identical point mutation.

\section{Methods}

Materials. ${ }^{35}$ S $]$ dATP was purchased from Amersham Corp. (Arlington Heights, IL). Random hexamer primers $\left[p d(N)_{6}\right]$ and dNTPs (dATP, dCTP, dGTP and dTTP) were from Pharmacia LKB Biotechnology (Piscataway, NJ). Taq DNA polymerase was procured from Perkin-Elmer Cetus (Norwalk, CT). Cell culture materials were obtained from Gibco Laboratories (Grand Island, NY).

Source of the cells and culture method. The source of eight of the MCAD-deficient cells has previously been reported (9). The ninth cell line was recently identified at Philadelphia. Eight MCAD-deficient cell 
lines were from unrelated U. S. Caucasians; YH1426 was from Spain. Two normal cell lines (GM05756, GM05565) were purchased from the NIGMS Human Genetic Mutant Cell Repository (Camden, NJ). The three other normal cell lines were obtained at Yale. In addition, two SCAD-deficient (10) and an isovaleric acidemia line (11) were used as controls. Cells were grown in MEM supplemented with $10 \%$ fetal calf serum and kanamycin at $37^{\circ} \mathrm{C}$ in $5 \% \mathrm{CO}_{2}$ atmosphere.

Isolation of DNA and RNA, and CDNA synthesis. Total cellular DNA and RNA were isolated from cells using the guanidium isothiocyanate method (12). First-strand cDNA was synthesized from total RNA using Moloney murine leukemia virus reverse transcriptase and a cDNA synthesis kit (Bethesda Research Laboratories, Gaithersburg, $\mathrm{MD}$ ) as follows: annealing was performed by mixing $10 \mu \mathrm{g}$ of total RNA with $5 \mu \mathrm{g}$ of $\operatorname{pd}(\mathrm{N})_{6}$ and incubating at $70^{\circ} \mathrm{C}$ for $5 \mathrm{~min}$. Transcription was carried out at $37^{\circ} \mathrm{C}$ for $90 \mathrm{~min}$ in $50 \mu \mathrm{l}$ of reaction mixture containing $50 \mathrm{mM}$ Tris- $\mathrm{HCI}, \mathrm{pH} 8.3,75 \mathrm{mM} \mathrm{KCI}, 3 \mathrm{mM}$ $\mathrm{MgCl}_{2}, 10 \mathrm{mM}$ DTT, $0.5 \mathrm{mM}$ dNTPS, and $2.5 \mu \mathrm{l}$ of reverse transcriptase.

PCR amplification of human PMCAD cDNA and genomic DNA. PCR amplification was carried out using 8-10 $\mu$ l of the first-strand cDNA synthesis mixture as the template and $1 \mu \mathrm{M}$ each of an appropriate pair of the primers. The PCR reaction mixture contained 10 $\mathrm{mM}$ Tris-HCI, $\mathrm{pH} 8.3,1.5 \mathrm{mM} \mathrm{MgCl}_{2}, 50 \mathrm{mM} \mathrm{KCI}, 0.01 \%$ gelatin, $200 \mu \mathrm{M}$ dNTPs and $0.5 \mu \mathrm{l}$ of Taq DNA polymerase in $100 \mu \mathrm{l}$, and was covered with $100 \mu \mathrm{l}$ of mineral oil. 30 cycles of PCR reaction were performed using a DNA Thermal Cycler (Perkin-Elmer Cetus) according to the following program: $1 \mathrm{~min}$ denaturation at $94^{\circ} \mathrm{C}, 2 \mathrm{~min}$ annealing at $50^{\circ} \mathrm{C}$, and $3 \mathrm{~min}$ extension at $72^{\circ} \mathrm{C}$. The final extension was for $7 \mathrm{~min}$.

For the PCR amplification of genomic DNA sequence, $2 \mu \mathrm{g}$ of high molecular weight genomic DNA was used as template after boiling for $5 \mathrm{~min}$. Other conditions were the same as above except for $2 \mathrm{~min}$ extension at $72^{\circ} \mathrm{C}$.

Subcloning of PCR-amplified products. The PCR-amplified products were first digested with Bam HI or Xba I (Promega Biotec, Madison, WI), and purified by electrophoresis using $1.5 \%$ low-melting agarose gel (SeaPlaque; FMC BioProducts, Rockland, ME). The fragments of expected size were cut out, extracted with phenol/chloroform, precipitated with ethanol, and subcloned into pBluescript vector (Stratagene Corp., La Jolla, CA). The recombinant plasmid was amplified by transforming Escherichia coli, strain XL1-Blue (Stratagene).
DNA sequencing. DNA sequencing was performed by the dideoxysequencing method (13) using $\left[{ }^{35} \mathrm{~S}\right] \mathrm{dATP}$ and Sequenase kit (United States Biochemical Corp., Cleveland, $\mathrm{OH}$ ).

Digestion of PCR products with Nco I. The PCR-amplified 87-bp fragment, encompassing A-955 to A-1041, was extracted and suspended in $20 \mu \mathrm{l} \mathrm{H}_{2} \mathrm{O} .10-\mu \mathrm{l}$ aliquots were digested with $5 \mathrm{U}$ of Nco I (Boehringer Mannheim Biochemicals, Indianapolis, IN) at $37^{\circ} \mathrm{C}$ for 2 $\mathrm{h}$, and the products were electrophoresed on $4 \%$ agarose gel (NuSieve GTG; FMC BioProducts) containing ethidium bromide.

\section{Results}

Amplification and sequences of variant pMCAD mRNA using $P C R$. We amplified the entire pMCAD cDNA coding region plus some untranslated flanking regions in two overlapping sections using two sets of 27 mer primers. In order to introduce appropriate restriction sites (Bam HI or Xba I), we artificially substituted one to three nucleotides in each primer. The expected sizes of the $5^{\prime}$ and $3^{\prime}$ fragments were 520 and $870 \mathrm{bp}$, encompassing positions -17 to 502 and positions 443 to 1312 , respectively.

The size of the major band in each of the $5^{\prime}$ and $3^{\prime}$ fragments amplified from cDNAs from a normal (GM05756) and three MCAD-deficient cells (YH1475, YH1476, and YH1426) was exactly as predicted (data not shown). Each fragment was subcloned and sequenced. In all six clones of the $3^{\prime}$ fragment from YH1475, G was found at position 985 in the pMCAD sequence, whereas in the corresponding fragment from the normal cell line and in the previously published normal human liver pMCAD sequence (7), this position is occupied by an $\mathbf{A}$ (Fig. 1). This $\mathbf{A} \rightarrow \mathbf{G}$ transition alters Lys-329 to Glu in pMCAD. Assuming that the leader peptide cleavage site in human pMCAD is the same as in rat pMCAD (8), this residue corresponds to residue 304 in the mature human MCAD. Likewise, in six and two clones of the $3^{\prime}$ fragment of cDNA from YH1426 and YH1476, respectively, the same A $\rightarrow$ G-985 transition was identified (data not shown).

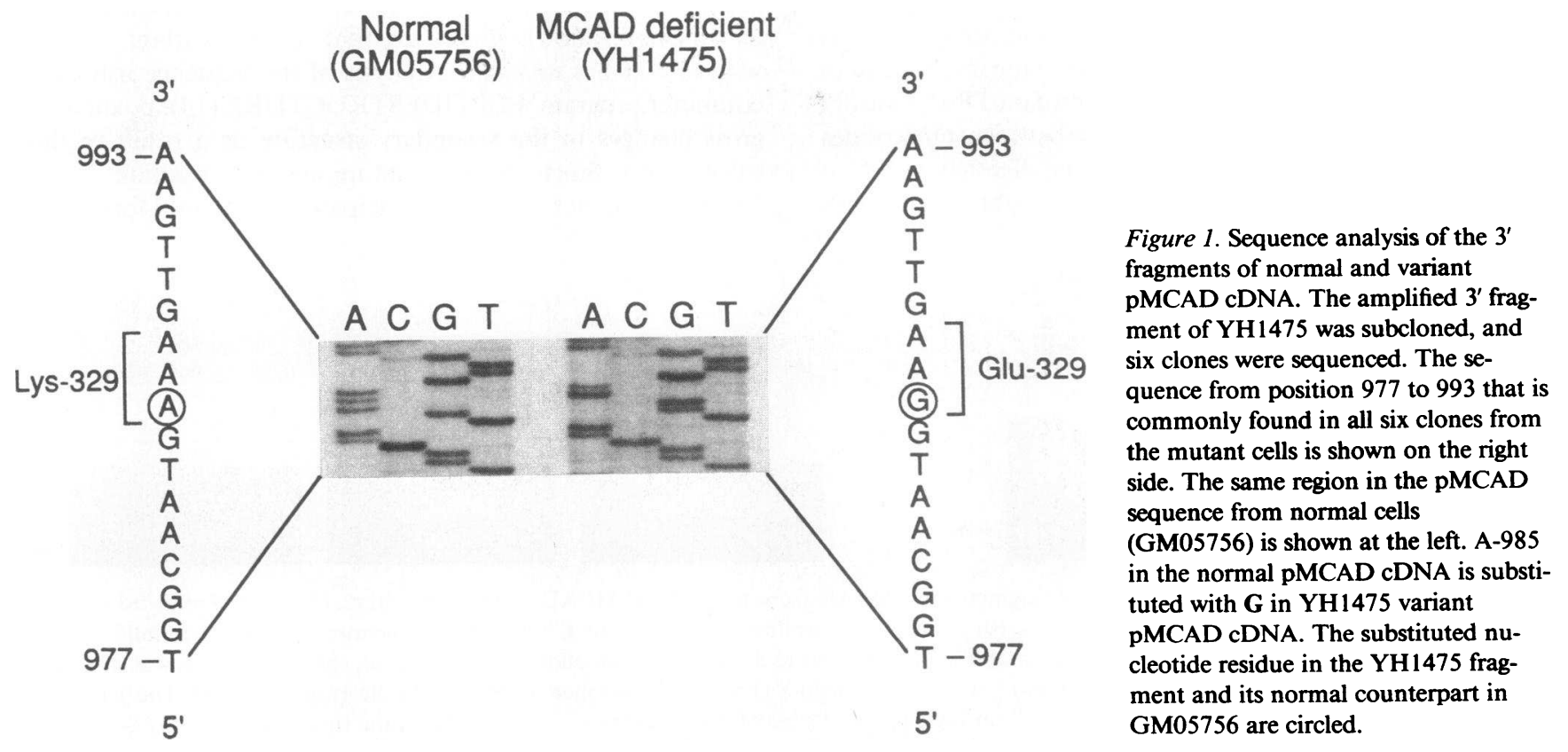




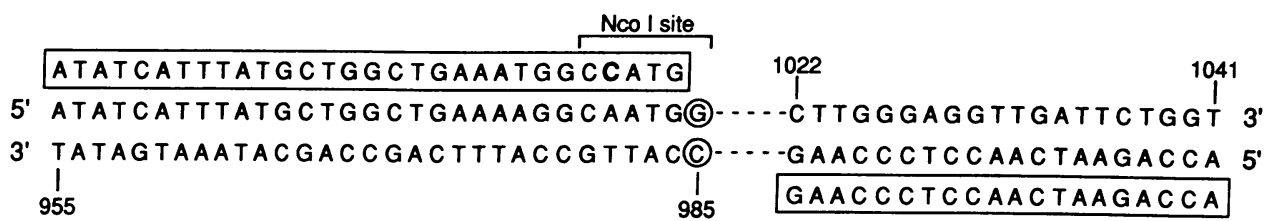

Figure 2. Strategy for PCR amplification that introduces an Nco I site at the mutation site in the variant sequence. In the second and third lines, the anti-sense and sense strand sequences, respectively, of mutant cDNA from position 955 to

985 and that from 1022 to 1041 are shown. $G$ and $C$ in the circle indicate the substituted nucleotides. The nucleotide sequences in the rectangles indicate a pair of primers, a 30 mer and a 20 mer, that were used to amplify the section of pMCAD cDNA from position 955 to 1041 and the corresponding section of genomic DNA. Note that in the upstream 30 mer primer, a mismatch, shown with a bold C, is introduced at position 981 to produce an Nco I site in the mutant fragments. The expected size of the PCR-amplified fragments is 87 bp.

Identification of $\boldsymbol{A} \rightarrow \mathbf{G}-985$ transition in nine $M C A D$-deficient cells using Nco I digestion. Since the nature of the variant MCAD protein in MCAD-deficient patients is known to be uniform (9), we devised a simple screening method for the identification of the $\mathbf{A} \rightarrow \mathbf{G - 9 8 5}$ transition that involves restriction enzyme digestion. Neither the normal nor mutant sequence surrounding the $A \rightarrow$ G-985 transition contained any restriction sites that could be used for segregating the normal and variant sequences. However, an Nco I site could be introduced to the mutant sequence by substituting A-981 with a $\mathrm{C}$ in the anti-sense strand (Fig. 2). Incorporating this feature, we designed a pair of PCR primers. The upstream primer was a 30 mer encompassing A-955 to G-984 in the anti-sense strand, in which the $A$ to $C$ substitution at position 981 was artificially introduced. The downstream primer was a 20 mer with a normal sequence starting from G-1022 in the sense strand (Fig. 2). Transcription of the anti-sense strand starts right from position 985 , incorporating $A$ by reading the normal template or $\mathrm{G}$ by reading the variant template.

The size of the major band that was amplified from cDNA from two normal (GM05565 and GM05756) and three MCAD-deficient cell lines (YH1475, YH1476, and YH1416) was 87 bp as expected (Fig. $3 A$ ). When digested with Nco I, the 87-bp copy of normal cDNAs remained intact, but that from the three MCAD-deficient cells completely disappeared, producing 61- and 26-bp bands (Fig. $3 \mathrm{~A}$ ). Likewise, the same cDNA copies from six additional MCAD-deficient cell lines were all cleaved by Nco I. In contrast, the copies from additional six control cDNAs remained intact (data not shown).

To confirm that the $A \rightarrow$ G-985 transition is intrinsic to the MCAD-deficient cells, PCR amplification was performed using genomic DNA as template. The size of the major band in the amplified copies of all normal and mutant DNAs was 87 $\mathrm{bp}$, indicating that at least the segment between nucleotides 985-1021 is located in a single exon. When digested with Nco I, the 87-bp fragments amplified from all eight control cells remained intact, whereas those from all nine MCAD-deficient cells were cleaved, producing 61 - and 26-bp pieces (Fig. $3 \mathrm{~B}$ ). In the amplification product of the $\mathrm{YH} 1426$ gene, there was an additional major band, estimated to be $94 \mathrm{bp}$. Although it was not as prominent, this 94-bp band was also detected in the amplification product from YH1426 cDNA. In initial sequencing experiments, all six clones of 870-bp $3^{\prime}$ fragments that were amplified from this cDNA contained $A \rightarrow$ G-985. Thus, it is likely that the 94-bp band is a PCR artifact. These data suggest that YH1426 is also homozygous for this point mutation.

\section{Discussion}

The results presented here disclosed a highly unusual feature of genetic MCAD deficiency, namely that an A $\rightarrow$ G-985 transition was found in all nine MCAD-deficient patients tested so far. Furthermore, all of them were homozygous for this point mutation, indicating that all 18 variant MCAD alleles in these cells carry this point mutation. Thus, it is very likely that the A $\rightarrow$ G-985 transition is the single prevalent mutation in the human MCAD gene causing MCAD deficiency in Caucasians. The incidence of this mutation in other ethnic groups needs to be studied.

When MCAD-deficient cells were incubated with $\left[{ }^{35}\right.$ S $]$ methionine, variant MCAD was strongly labeled (9). However, recent experiments indicate that, unlike MCAD in normal cells, variant MCAD could not be detected by Western blot analysis in any of the MCAD-deficient cell lines with this point mutation (Coates, P. M., Y. Indo, and K. Tanaka, unpublished observation), suggesting that mature variant MCAD with Glu-304 is unstable. Analysis of the sequence using the computer program, PEPTIDESTRUCTURE (14), predicts no gross changes in the secondary structure as a result of this substitution. Since in each mature subunit, position 304 is located in the domain that participates in tetramer formation
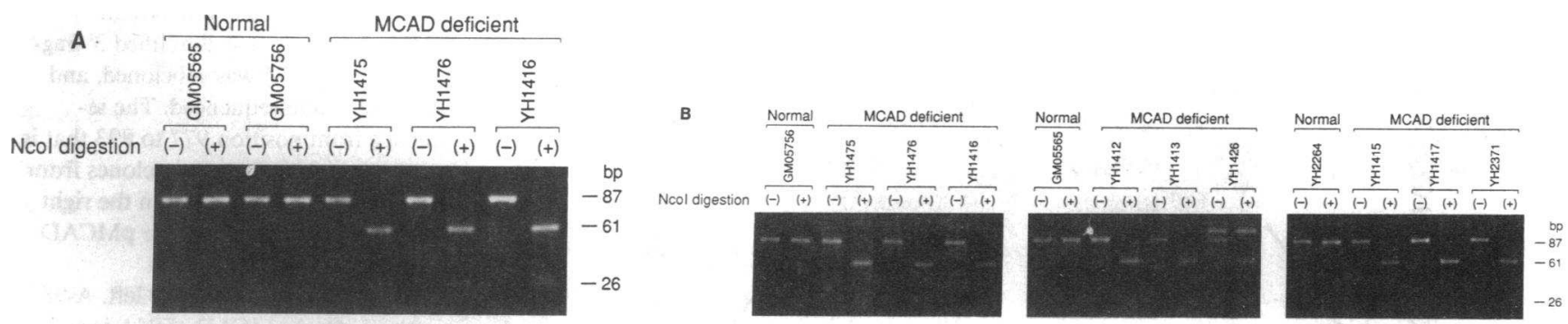

Figure 3. Nco I digestion of the PCR-amplified fragments of pMCAD from normal and MCAD-deficient cell lines. $(A)$ Experiments using fragments amplified from $\mathrm{CDNA}$, and $(B)$ experiments using fragments amplified from genomic DNA. cDNA or genomic DNA was amplified using the pair of primers shown in Fig. 2 and the amplified products were divided into two aliquots. One aliquot, shown with (-), was directly applied to the gel. The other aliquot, shown with $(+)$, was digested with $5 \mathrm{U}$ of Nco I, and then applied to the electrophoretic gel. The gel used was $4 \%$ NuSieve GTG agarose gel containing ethidium bromide. The sizes of digested products are shown on the right side. 
(15), we speculate that the substitution of a basic residue in this position with an acidic one may inhibit tetramer formation, leading to instability of the enzyme.

The observation in patients with MCAD deficiency is in sharp contrast to the findings for variant IVD in patients with isovaleric acidemia and variant SCAD in patients with SCAD deficiency. Among 15 isovaleric acidemia patients, at least five distinct types of variant alleles were recognized with regard to the presence and absence of variant IVD proteins and their molecular size (11). In three patients with SCAD deficiency, variant SCAD was of normal size as tested with $\left[{ }^{35}\right.$ S]methionine labeling and immunoprecipitation (16). However, the results from immunoblot analysis and pulse-chase experiments indicate that one of the variant SCADs was unstable, resulting in cross-reacting materials (CRM)-negativity while two others were stable and CRM positive (10). Subsequent sequence analysis demonstrated that the CRM-negative SCAD-deficient cells contained two distinct mutant alleles, each encoding a different point mutation (17).

Apart from the interest in the genetic origin of $\mathbf{A} \rightarrow \mathbf{G - 9 8 5}$, our present results are clinically significant. The accurate and prompt diagnosis of MCAD deficiency is important because of its relatively high incidence and unpredictable clinical manifestations. However, it was difficult until recently, since the currently available enzyme assay methods are cumbersome and time consuming. Recently, the stable isotope dilution/ mass spectrometric method for urinary acylglycines has made it possible to make a prompt and accurate diagnosis of this disease (18). However, when tested in complete remission, a small number of MCAD-deficient patients present borderline values of these compounds. The identification of the $\mathbf{A} \rightarrow$ G-985 transition using Nco I digestion, possibly in conjunction with the use of dried blood on filter paper as the source of DNA (19), offers an excellent confirmatory diagnostic method for this elusive disease.

Note added in proof: Our subsequent sequence study indicates that the longer PCR fragment in YH1426 contains a 13-bp tandem repeat from position 999 , causing a premature stop codon at the $5^{\prime}$ end of the repeat. However, the 985th nucleotide was $A$ as in the wild type. Hence, this band represents a second variant allele, and YH1426 is a compound heterozygote. This variant MCAD gene appears to be not effectively transcribed, or alternatively, it produces unstable mRNA.

\section{Acknowledgments}

We acknowledge that the enzyme diagnosis in the MCAD-deficient patients reported here was previously established by Dr. Daniel E. Hale. We thank Mrs. Connie Woznick for preparing this manuscript.

This work was supported by grants from the National Institutes of Health (DK-38154 and NS-17752).

\section{References}

1. Kolvraa, S., N. Gregersen, E. Christensen, and N. Hobolth. 1982. In vitro fibroblast studies in a patient with $\mathrm{C}_{6}-\mathrm{C}_{10}$-dicarboxylic aciduria: evidence for a defect in general acyl-CoA dehydrogenase. Clin. Chim. Acta. 126:53-67.

2. Roe, C. R., and P. M. Coates. 1988. Acyl-CoA dehydrogenase deficiencies. In The Metabolic Basis of Inherited Disease. C. R. Scriver, A. L. Beaudet, W. S. Sly, and D. Valle, editors. McGraw Hill Inc., New York. 889-914.

3. Duran, M., M. Hofkamp, W. J. Rhead, J. M. Saudubray, and S. K. Wadman. 1986. Sudden infant death and "healthy" affected family members with medium chain acyl-CoA dehydrogenase deficiency. Pediatrics. 78:1052-1057.
4. Ikeda, Y. Y., K. Okamura-Ikeda, and K. Tanaka. 1985. Purification and characterization of short chain, medium chain, and long chain acyl-CoA dehydrogenases from rat liver mitochondria: isolation of the holo- and apoenzymes and conversion of the apoenzyme to the holoenzyme. J. Biol. Chem. 260:1311-1325.

5. Matsubara, Y., Y. Indo, E. Naito, H. Ozasa, R. Glassberg, J. Vockley, Y. Ikeda, J. P. Kraus, and K. Tanaka, 1989. Molecular cloning and nucleotide sequence of cDNAs encoding the precursors of rat long chain acyl-coenzyme $A$, short chain acyl-coenzyme $A$ and isovaleryl-coenzyme A dehydrogenases: sequence homology of four enzymes of the Acyl-CoA dehydrogenase family. J. Biol. Chem. 264:16321-16331.

6. Ikeda, Y., S. M. Keese, W. A. Fenton, and K. Tanaka. 1987. Biosynthesis of rat liver mitochondrial short chain acyl-CoA, medium chain acyl-CoA, long chain acyl-CoA, and isovaleryl-CoA dehydrogenases: in vitro synthesis, import into mitochondria, and processing of their precursors in a cell-free system and in cultured cells. Arch. Biochem. Biophys. 252:662-674.

7. Kelly, D. P., J.-J. Kim, J. J. Billadello, B. E. Hainline, T. W. Chu, and A. W. Strauss. 1987. Nucleotide sequence of medium-chain acylCoA dehydrogenase mRNA and its expression in enzyme-deficient human tissue. Proc. Natl. Acad. Sci. USA. 84:4068-4072.

8. Matsubara, Y., J. P. Kraus, H. Ozasa, R. Glassberg, G. Finocchiaro, Y. Ikeda, J. Mole, L. E. Rosenberg, and K. Tanaka. 1987. Molecular cloning and nucleotide sequence of cDNA encoding the entire precursor of rat liver medium chain acyl coenzyme A dehydrogenase. J. Biol. Chem. 262:10104-10108.

9. Ikeda, Y., D. E. Hale, S. M. Keese, P. M. Coates, and K. Tanaka. 1986. Biosynthesis of variant medium chain acyl-CoA dehydrogenase in cultured fibroblasts from patients with medium chain acyl-CoA dehydrogenase deficiency. Pediatr. Res. 20:843-847.

10. Naito, E., Y. Indo, and K. Tanaka. 1989. Short chain acylcoenzyme A dehydrogenase (SCAD) deficiency. Immunochemical demonstration of molecular heterogeneity due to variant SCAD with differing stability. J. Clin. Invest. 84:1671-1674.

11. Ikeda, Y., S. M. Keese, and K. Tanaka. 1985. Molecular heterogeneity of variant isovaleryl-CoA dehydrogenase from cultured isovaleric acidemia fibroblasts. Proc. Natl. Acad. Sci. USA. 82:7081-7085.

12. Davis, L. G., M. D. Dibner, and J. F. Battey. 1986. Basic Methods in Molecular Biology. Elsevier Science Publishing Co., New York. 130-135.

13. Sanger, F., S. Nicklen, and A. R. Coulson. 1977. DNA sequencing with chain-terminating inhibitors. Proc. Natl. Acad. Sci. USA. 74:5463-5467.

14. Devereux, J., P. Haeberli, and O. Smithies. 1984. A comprehensive set of sequence analysis programs for the VAX. Nucleic Acids Res. 12:387-395.

15. Kim, J.-J. P., and J. Wu. 1988. Structure of medium-chain acyl-CoA dehydrogenase from pig liver mitochondria at $3-\AA \AA \AA$ resolution. Proc. Natl. Acad. Sci. USA. 85:6677-6681.

16. Naito, E., H. Ozasa, Y. Ikeda, and K. Tanaka. 1989. Molecular cloning and nucleotide sequence of complementary DNAs encoding human short chain acyl-coenzyme A dehydrogenase and the study of the molecular basis of human short chain acyl-coenzyme A dehydrogenase deficiency. J. Clin. Invest. 83:1605-1613.

17. Naito, E., Y. Indo, and K. Tanaka. 1990. Identification of two variant short chain acyl-coenzyme A dehydrogenase alleles, each containing a different point mutation in a patient with short chain acylcoenzyme A dehydrogenase deficiency. J. Clin. Invest. 85:1575-1582.

18. Rinaldo, P., J. J. O'Shea, P. M. Coates, D. E. Hale, C. A. Stanley, and K. Tanaka. 1988. Diagnosis of medium chain acyl-CoA dehydrogenase deficiency by stable isotope dilution analysis of urinary n-hexanoylglycine and 3-phenylpropionylglycine. N. Engl. J. Med. 319:1308-1313.

19. McCabe, E. R. B., S. Z. Huang, W. K. Seltzer, and M. L. Law. 1987. DNA microextraction from dried blood spots on filter paper blotters: potential applications to newborn screening. Hum. Genet. 75:213-216. 